Meta

Journal des traducteurs

Translators' Journal

\title{
Les fondements de l'organisation d'un dictionnaire d'usages
}

\section{Francisco Da Silva Borba}

Volume 41, numéro 2, juin 1996

Traduction et terminologie au Brésil

Translation and Terminology in Brazil

URI : https://id.erudit.org/iderudit/002102ar

DOI : https://doi.org/10.7202/002102ar

Aller au sommaire du numéro

Éditeur(s)

Les Presses de l'Université de Montréal

ISSN

0026-0452 (imprimé)

1492-1421 (numérique)

Découvrir la revue

Citer cet article

Borba, F. D. S. (1996). Les fondements de l'organisation d'un dictionnaire d'usages. Meta, 41(2), 279-287. https://doi.org/10.7202/002102ar

\section{Résumé de l'article}

L'auteur discute de l'organisation d'un dictionnaire d'usages (D.U.). Comment l'idéologie des auteurs influence-t-elle l'information contenue dans un D.U. ?; autour de quels objectifs le D.U. s'organise-t-il?; quels critères choisir pour établir la nomenclature? ; qu'en est-il de l'information grammaticale et quel est son rôle dans un D.U. ? 


\title{
LES FONDEMENTS DE L'ORGANISATION D'UN DICTIONNAIRE D'USAGES
}

Francisco Da SiLVA Borba

São Paulo

\begin{abstract}
Résumé
L'auteur discute de l'organisation d'un dictionnaire d'usages (D.U.). Comment l'idéologie des auteurs influence-t-elle l'information contenue dans un D.U.?; autour de quels objectifs le D.U. s'organise-t-il?; quels critères choisir pour établir la nomenclature?; qu'en est-il de l'information grammaticale et quel est son rôle dans un D.U.?
\end{abstract}

\begin{abstract}
The author addresses a number of issues regarding the conception of dictionaries of general usage: How does the author's ideology influence content? What should the objectives of such dictionaries be? What should the criteria for establishing nomenclature be? What kind of grammatical information should they provide, and for what purpose?
\end{abstract}

Un dictionnaire d'usages (D.U.) est un dictionnaire de langue dans la mesure où il s'occupe de signes linguistiques. Un dictionnaire qui compte saisir le signe en mouvement doit se rapporter aux trois dimensions du langage - syntaxique, sémantique et pragmatique - ce qui se fait sur une base théorique éclectique. S'il veut être un guide d'usages, il doit, en principe, prendre la forme d'un ensemble d'instructions (syntaxiques, sémantiques, pragmatiques) dont les détails et la rigueur peuvent compliquer la sélection et l'ordre des informations, au risque, tout de même, de dépasser le cadre de l'ouvrage.

Étant donné que les dictionnaires ont une influence considérable sur le public, ils constituent un outil idéologique très puissant. L'idéologie, en orientant les convictions et en traduisant les attitudes et les attentes de l'auteur, est toujours présente - soit pour les décisions sur les objectifs qui justifieront le choix des entrées, soit pour la sélection et la rédaction des informations internes, dans la mesure où personne n'informe sans partialité ; au contraire, on veut souvent expliquer, persuader, prescrire ou instruire. On comprend, ainsi, que l'engagement socioculturel du dictionnaire est plus grand que celui de la grammaire, parce que son rapport avec la culture est très étroit.

Si l'idéologie est déjà engagée à l'identification et au règlement des desseins et, si, pour un D.U., le but pédagogique est placé en premier lieu, c'est parce qu'il présuppose que l'éducation linguistique est le fondement de n'importe quel genre de progrès social, vu que c'est par le biais du langage qu'on analyse, qu'on reconnaît et qu'on domine le monde. Cette attitude détermine les informations contenues dans chaque entrée.

Dans la liste des exemples, l'idéologie oriente l'adoption d'une conception de la norme, qui s'applique soit au choix, soit à la représentation des différents niveaux de langue enregistrés (populaire, argot, technique).

L'usage, entendu comme quelque chose qu'on apprend à l'école, implique confiance et adresse dans le maniement du code écrit. Il est conditionné par l'adéquation ou par le rapport précis, claire et naturel entre forme et contenu.

Effectivement, l'éducation linguistique accomplie grâce à l'exercice des modèles écrits porte aussi sur le progrès de l'usage de la langue parlée (souplesse et aisance). 
C'est par là, peut-être, que se soulage un peu l'opacité de la parole, comme résultat de la valeur générique qu'on attribue aux syntagmes en l'absence du terme précis.

Un dictionnaire d'usages s'organise autour de trois objectifs: (i) pédagogique donner aux usagers un outil de contrôle sur les modèles écrits; (ii) théorique - identifier des relations grammaticales dans le lexique et/ou en vérifier la structure; (iii) méthodologique - tester un modèle de description grammaticale de la langue: une théorie argumentative selon le schéma $\mathrm{P}(\mathrm{A})$.

Les propos ci-dessus conditionnent la macro et la microstructure du dictionnaire dont la forme est déterminée par une théorie grammaticale donnée.

On établit la nomenclature d'un dictionnaire à partir de trois critères: morphologique, psychologique et de fréquence. Le critère morphologique fournit tout un réseau d'éléments généré par dérivation. Comme pour l'élaboration des dictionnaires dits extensionnels (ex.: le Petit Robert). À son tour, la psycholinguistique peut être utile pour l'organisation des dictionnaires plus orientés, dans la mesure où ils fournissent des pistes pour établir les priorités des zones lexicales dans l'apprentissage aux groupes professionnels, au développement de la scolarité. Malheureusement, la psycholinguistique ne s'est pas dévéloppée suffisamment pour nous fournir des renseignements précis sur les mécanismes d'assimilation et de développement des expédients grammaticaux ou sur les stratégies de perception de la langue maternelle.

Le critère de fréquence peut être un bon principe de mesure objective du lexique. Il permet d'identifier le vocabulaire de base et l'établissement d'échelles lexicales, ce qui a son importance lorsqu'il s'agit de choisir les unités qui serviront pour les définitions, les explications, etc. La fréquence peut être utile encore pour trouver une moyenne d'usage (ou usage moyen), sans perdre de vue une difficulté fondamentale, celle de ne refléter que la nature des textes analysés, qui changent selon l'époque, le lieu, le niveau de langue, le véhicule (langue parlée, langue écrite), le sujet, l'éducation linguistique, etc.

Une autre difficulté se rapporte à l'étendue des textes et aux nombreuses interprétations qu'on leur donne, ainsi qu'à la conception des mots qu'on utilise.

Pour aboutir à l'usage moyen, il faut d'abord considérer le rapport mot courant/mot technique, étant donné qu'on assiste aujourd'hui à une sorte de survalorisation des technologies: le vocabulaire technique se répand très vite.

Mais il faut encore considérer deux points de vue: (i) le terme technique se répand dans un domaine et par rapport aux zones voisines; (ii) les mots tendent à se disséminer et à s'introduire dans la langue commune, le métier étant l'activité sociale la plus importante.

Le vocabulaire d'une communauté quelconque est, d'habitude, marqué par ses activités professionnelles dominantes (les termes se rapportant, par exemple, à la vie maritime pour le portugais).

Si la nomenclature d'un D.U. est établie par un critère de fréquence, on devra suivre les étapes ci-dessous:

1- établissement des concordances ad hoc, c.-à-d., dépouillement des occurrences dans leurs contextes;

2- réduction des contextes fournis par l'ordinateur à des contextes pertinents, ou bien à ceux dans lesquels on a des mots jouant un rôle actif de sélection sur le mot à décrire;

3- établissement des paradigmes d'acception et étude des rapports distributionnels entre distribution et signification, en considérant les traits sémantiques des cooccurrents du mot central ;

4 réordination des contextes à des ensembles différents, par les traits sélectifs syntaxiquessémantiques qui les identifient. Positionnement relatif de chaque sous-ensemble en fonction de la fréquence observée dans le corpus; 
5- lemmatisation des formes;

6- établissement du nombre d'entrées par le degré de détachement syntaxiquesémantique observé parmi les sous-ensembles et, aussi, selon les indices fréquentiels;

7 - standardisation des entrées. Intégrations des ensembles de mots. Choix de contextes pour les exemples illustratifs.

La fréquence brute peut être relativisée par le biais d'un critère lexico-statistique, qui permet: (i) de constater les priorités structurales; (ii) de déceler si le mot a des préférences pour une fonction syntaxique quelconque: sujet, complément, prédicat, etc. Si oui, il faut en identifier la motivation, peut-être sa structure conceptuelle, ses rapports distributionnels, ou encore, la structure de la phrase où il se trouve; (iii) permet également de déceler quels sont les mots qui «cooccurrent» le plus aisément (pour aboutir à des syntagmes figés (ex. : remonter au déluge)).

En présupposant qu'il n'y a pas de mot à distribution neutre, on établit: (i) qu'un mot en appelle un autre; (ii) que certains mots sont plus orientés que d'autres vers certaines fonctions syntaxiques (ex.: les noms abstraits comme noyau de prédicat après certains verbes - v. supra). On constate cela en observant le corpus de référence après l'inventaire des formes, ou bien en préparant une liste de mots qu'on présente aux usagers pour qu'ils fassent des phrases, ce qui sert à tester leurs réactions et leurs tendances. On aura affaire, par exemple, aux traits syntaxiques-sémantiques qui privilégient quelques fonctions (cf. +anim ou thum pour la fonction de sujet), ou les stéréotypes motivés par la culture (ex. : l'apogée de Rome).

Une analyse lexico-statistique fournit les voies pour aboutir à l'échelle opératoire des concepts de structure permanente et de structure accidentelle. La structure permanente est une structure à haute fréquence, stable, celle qui vient à l'esprit en premier lieu parce qu'elle est peu sensible aux interférences. Elle comprend des associations ou des combinatoires spontanées et simples: le chien aboie; l'assiette s'est cassée. D'autre part, la structure accidentelle est plus rare, peu stable et plus soumise à des interférences, ayant un faible niveau d'organisation. Ce sont de nouvelles structures conceptuelles créées par l'usager pour répondre à des situations originales (l'innocente beauté des jardins et du jour (La Fontaine)). Ex. :

\section{1. (a) Le coiffeur travaille avec ses ciseaux}

(b) Le coiffeur pétrit des cerises avec une fourchette

Structure permanente

Structure accidentelle

Les structures permanentes engendrent des syntagmes figés et, par là, des stéréotypes. En principe, on s'attend à trouver des structures permanentes dans les textes scientifiques, et des structures accidentelles dans les textes littéraires. Le jeu de ces deux types détermine la dynamique de la communication et le statut d'adéquation (propriété, économie, clarté).

On présuppose que les structures permanentes ont toujours une forme typique. Donc, la recherche sur l'usage devrait se concentrer surtout sur les structures accidentelles.

L'usage adroit des structures accidentelles se rapporte non seulement à la connaissance du monde et à l'aisance de l'usager à percevoir, à accepter, à incorporer et à discerner les mondes possibles, mais aussi à la facilité d'usage de la langue, c'est-à-dire à son éducation linguistique.

Pour établir l'ensemble des entrées par le critère fréquentiel, on a deux variables: la fréquence relative des éléments par couche sociale ou par région, et l'intersection de ces derniers d'une couche à l'autre ou d'une région à l'autre. J'ai déjà souligné qu'il s'agit de 
chercher l'usage moyen (que l'école stimulerait ou transmettrait), sans qu'on ait nécessairement un intérêt spécifique à établir un dépouillement exhaustif de plusieurs couches ou de points du territoire national: pour la langue écrite, ce niveau n'est pas un guide fiable pour des considérations d'ordre sociolinguistique, étant donné la migration des mots et l'instabilité de la langue écrite comme marque régionnale. Et pourtant, autant que possible, les chaînes devront être identifiées par couches (familier, littéraire, technique, argotique, etc.) et par région (nord, sud, est...), qui sont deux étiquettes s'opposant au discours considéré comme neutre. Même partiellement, l'annotation des couches peut servir de piste à qui veut étudier (observer, analyser) les réactions verbales ou les attitudes des usagers envers le discours qu'ils emploient eux-mêmes.

À ce sujet, je suis d'accord avec Dubois, quand il déclare que le dictionnaire est un miroir où l'usager se reconnaît comme locuteur natif et comme participant d'une culture - il y trouve soit la confirmation de ses jugements de grammaticalité (qui définit le degré de correction des phrases), soit la confirmation de ses jugements d'acceptation, qui définissent son appartenance à une culture déterminée.

Au Brésil, on a tendance à considérer le lexique comme un ensemble d'unités qui peut être décrit isolément, étant donné qu'il n'est qu'un amas d'informations apprises; autrement dit, il n'est qu'une liste d'unités à informations mémorisables. La créativité est vue comme le produit des règles de structure des phrases et des transformations. L'aisance de l'usager à entendre/produire des phrases nouvelles se borne à la connaissance qu'il a des règles de production lui permettant de combiner, à l'infini, l'ensemble fixe des mots mémorisés.

Mais, d'autre part, le lexique n'est pas un amas d'unités isolées parce que: (i) il est aussi une force créative, dans la mesure où il $\mathrm{y}$ a un jeu productif et ouvert de formation des mots: de nouvelles formations morphématiques apparaissent à tout venant (il suffit qu'on en ait besoin); (ii) si la syntaxe s'occupe de toute combinatoire lexicale, le corollaire évident est que chaque mot a un statut syntaxique.

Par conséquent, le lexique ne doit pas être vu comme un simple inventaire qui spécifie les formes et les sens des unités : un élément lexical doit ĉtre décrit par rapport à ses propriétés phoniques, syntaxiques, sémantiques et pragmatiques.

Un dictionnaire qui oriente (explique, suggère, contrôle) l'usage aura obligatoirement une base grammaticale. Cela ne signifie pas qu'il s'agit du seul critère pour décider du travail des lexicographes. Une première tâche, préliminaire et fondamentale à celui qui a un intérêt pédagogique, est de savoir dans quelle mesure (et probablement dans quelle direction) les mots circulent. Combien de mots y a-t-il dans la langue écrite d'aujourd'hui ? Quel est le rapport entre un élément et son occurrence, hors la séparation grossière et élémentaire qui se fait entre unités grammaticales (les plus fréquentes)? De cette façon, la première tâche est d'identifier le degré de répétition lexicale de la langue écrite, entendue comme relation simple entre le signe et son occurrence.

Même pour le portugais, ce sujet est encore vierge. D'après Richman-Carrol \& Davies - American Heritage Word Frequency Book - 5088720 occurrences recueillies fournissent 86741 mots distincts.

Un D.U. doit tout faire pour stimuler l'intuition du locuteur natif. Un des moyens sera de montrer comment deux éléments dérivent l'un de l'autre: si l'on connaît l'un, il devient plus facile d'apprendre l'autre (ex. : proliférer - prolifération). Une grammaire révélant ces faits aurait une meilleure évaluation qu'une qui ne les révèlerait pas. Le fondement grammatical sous-jacent à l'élaboration d'un D.U., que j'appelle lexicogrammaire, est donc une position théorique dont le but est d'arriver aux propriétés syntaxiques du lexique, ce qui présuppose, au départ, que la syntaxe se place avant la sémantique, ou, alors, que la sémantique est une conséquence de la syntaxe. Ainsi, on conçoit les entrées du diction- 
naire comme des phrases simples et non pas comme des mots isolés. Conçue comme un ensemble de règles d'organisation du lexique, la lexicogrammaire permet de sélectionner les informations et de structurer les entrées visant un usage approprié.

Je ne pense évidemment pas que les informations grammaticales doivent être intégrées au dictionnaire même, et encore moins les entrées typiquement grammaticales: verbe, adjectif, pronom. Je pense plutôt à une orientation syntaxique (règles distributionnelles et transformationnelles), sémantique (règles de combinatoire des traits) et pragmatique (règles contextuelles et situationnelles). Par exemple: le choix des éléments qui doivent entrer à titre d'informations pertinentes pour chaque entrée: définition, synonymie, équivalence, explication. La définition constitue une chaîne syntaxique complète : le triangle est une figure géométrique à trois côtés.

Quant aux synonymes et aux équivalents, il faut être prudent car l'équivalence syntaxique n'est pas toujours la conséquence de l'équivalence sémantique ou de la relation synonymique.

Pour bien saisir cette position, il faut d'abord considérer les rapports entre grammaire et lexique comme des composants de la structure de la langue et, ensuite, appréhender la grammaire et le dictionnaire comme des outils descriptifs, c.-à-d. des produits de la recherche linguistique.

Le lexique, au même titre que la grammaire, intègre la structure linguistique, mais il ne constitue qu'une partie de cette structure; la grammaire fait aussi partie de cette dernière, mais considérée dans sa totalité. Il existe évidemment une base grammaticale et lexicale appartenant à tous les locuteurs, mais elle est différemment partagée. On dira que la grammaire internalisée est égale pour tous les usagers, mais que le lexique ne l'est pas, et ce, même en ce qui concerne les mots grammaticaux.

Il y a, en principe, un substrat commun à tous, ce qui rend plus aisée l'interaction sociale par le biais de la langue, et qui se constitue de mots grammaticaux et lexicaux choisis par la communauté comme fondamentaux. Chaque locuteur partage encore, en quelque sorte, les sous-lexiques en cours dans sa communauté, dans la mesure où il utilise et où il comprend le lexique régional et social aussi bien que le parler de ses camarades, de ses amis du même âge, etc.

Mais, pour des raisons diverses (ex.: structure mentale, motivation, éducation linguistique), cela se concrétise dans des proportions bien différentes. C'est pourquoi on dit qu'aucun usager ne domine le lexique de son voisin, et vice-versa. En fait, ce n'est pas pour les raisons présentées ci-dessus qu'il ne domine pas ce lexique, mais surtout du fait que ce dernier est indéterminé et possède un nombre très élevé d'unités. Il semble qu'un locuteur cultivé manipule un dixième du lexique total actif et passif de sa langue. La grammaire est intériorisée indépendamment des facteurs socioculturels, mais non le lexique - il n'est réel que pour desservir une communauté linguistique et, contrairement à la grammaire, il est indissolublement associé à une vision du monde. C'est par la grammaire que le locuteur accepte des phrases qu'il ne comprend pas : ce qui lui échappe, c'est le lexique. C'est par l'étendue et par l'usage du lexique actif et passif qu'on voit comment il est possible d'évaluer l'éducation linguistique. Du point de vue de l'apprentissage, les règles grammaticales de base sont intériorisées très tôt et une fois pour toutes; le lexique, non: étant donné qu'il est composite et renouvelable, son acquisition (ou expansion/rétraction) dure toute la vie. Malgré les différences lexicales, l'interaction linguistique reste possible car: (i) le vocabulaire de base est constitué par les mots les plus fréquents, rangés dans un ensemble homogène; (ii) la restriction lexicale est commune à presque tous les usagers; (iii) la vie en communauté impose des relations entre des locuteurs aux connaissances comparables. 
La grammaire est un ensemble fini de règles d'organisation et de fonctionnement de la langue; le dictionnaire est un ensemble d'unités lexicales listées indépendamment: il constitue un ensemble de morphèmes lexicaux, c'est-à-dire d'unités significatives codifiées, ou de signes. L'ensemble des entités grammaticales est fini, celui des éléments lexicaux constitue une liste ouverte: il est infini. Tant la signification des entités grammaticales est interne, tant celle des unités lexicales est externe. On peut aussi délimiter la portée de la grammaire et du dictionnaire par l'extension de la pertinence des modèles: tout ce qui s'applique à un nombre considérable d'éléments appartient à la grammaire; ce qui ne joue que pour une unité appartient au dictionnaire. Par exemple, le fait qu'un nombre considérable de mots est employé par paire - singulier et pluriel - justifie l'inclusion de ce phénomène dans la grammaire. De même pour les genres - masculin et féminin marqués par des morphèmes comme - $e$ (cf. lion/lionne; marchand/marchande). D'autre part, le fait qu'il y ait des paires où la différence de genre est indiquée par des mots différents, (homme/femme; bouc/chèvre; sanglier/laie) justifie qu'on les inclue dans le dictionnaire. Les mots sont consignés dans le dictionnaire par classes dont l'établissement relève de la grammaire, qui ne s'occupe pas des mots, mais des rapports entre les mots.

Bref, n'importe quelle information sur le fonctionnement de la langue appartient à la grammaire, mais l'information sur le fonctionnement d'une unité (qui, pourtant, se rapporte à la totalité), doit être cherchée dans le dictionnaire. Par exemple, si les règles flexionnelles sont déjà dans la grammaire, pourquoi le dictionnaire devrait-il renseigner l'usager sur la flexion de tel ou tel mot? Les règles syntaxiques générales sont dans la grammaire - accord, régimes, ordre, transformations. Donc, il n'est pas nécessaire de signaler comment chaque unité s'accorde avec une autre, puisque l'harmonie flexionnelle atteint toutes les unités.

Les fonctions générales — sujet, prédicat, complément — sont indiquées dans la grammaire avec les classes qui les remplissent. Donc, il est inutile de mentionner, à chaque entrée, si un nom ou un verbe fonctionne comme sujet ou complément ou comme noyau d'un prédicat. Mais si une sous-classe spéciale de V attire une sous-classe spécifique de $S$, cela relève du dictionnaire, puisque cela touche un élément particulier ou un groupe d'éléments.

La grammaire dit qu'une sous-classe $X$ de $P$ engage une sous-classe $Y$ de $S$, avec des traits; le dictionnaire accueille les éléments et les traits impliqués dans cette règle, et signale si cette combinatoire y ajoute des valeurs nouvelles ou différentes.

La grammaire liste les règles générales de dépendances, mais le régime particulier, bien que ses conséquences pour l'unité viennent dans le dictionnaire.

De cette façon, la séparation entre grammaire et dictionnaire semble radicale : celleci ne comporte pas d'unités lexicales, celui-là ne subit pas d'informations grammaticales. Quant au lexique, la grammaire devrait s'en tenir à des règles d'organisation et de fonctionnement, de même que le dictionnaire écarterait les formes liées et les informations phonétiques/ phonologiques, etc. Il se bornerait à lister les équivalences, les définitions, les explications. Et pourtant, ce radicalisme nous amènerait tout de suite à des impasses, comme par exemple la question de l'inclusion des mots grammaticaux.

À vrai dire, la différence entre grammaire et dictionnaire est plus pratique et méthodologique que conceptuelle. Voyons: personne ne critique la grammaire parce qu'elle contient des renseignements qui, en principe, seraient dans le dictionnaire (liste de mots, synonymes, homonymes, polysémie, antonymes, etc.), mais on dit aussi que les dictionnaires et les grammaires s'occupent souvent de choses égales d'ailleurs et, encore, que les dictionnaires donnent, pêle-mêle, des informations de nature purement grammaticale: des flexions (des féminins et des pluriels irréguliers, pluriels des noms composés, conjugaison des verbes irréguliers, etc.). Bref, considérons le lexique non pas comme une 
pure liste d'éléments indépendants, mais comme une composante du système linguistique et, ainsi, soumise à des règles d'organisation et de fonctionnement, c'est-à-dire à des règles de grammaire de la langue. D'autre part, le vocabulaire total ne compte pas seulement sur les unités lexicales en rapport direct avec la réalité extérieure, mais aussi sur des éléments grammaticaux, liste fermée, aisément identifiables et dont la valeur sémantique est dans le système linguistique lui-même. La fréquence même différencie les deux groupes: les unités grammaticales sont de très haute fréquence, les unités lexicales sont de fréquence inégale et variable car leur occurrence dépend de plusieurs facteurs - tous extérieurs au code linguistique.

Ainsi, c'est le mot lexical qui définit le mieux la nature du lexique en bâtissant un système de monde renfermé, et pourtant ce monde-là inclut une image de la langue, par le biais des mots métalinguistiques.

D'un point de vue pédagogique, on peut souligner que la grammaire et le lexique opèrent de façon indépendante : celle-ci à l'intérieur de la langue et celui-là au niveau de l'échange langue-réalité, en faisant la connexion nécessaire entre la langue et le monde extérieur.

C'est à ce niveau que les deux domaines ont des échelles différentes: on internalise un système de règles plus vite et plus complètement (parce qu'il est fini), c'est-à-dire qu'un locuteur quelconque sait reconnaître phrase et non-phrase. Personne ne domine le vocabulaire total de sa langue maternelle. Ainsi, la compétence lexicale optimale sera toujours définie d'une façon relative, car elle dépend de plusieurs facteurs (degré de scolarité, mémoire, métier). Par exemple, il y a des jugements de non-grammaticalité lexicale qui reposent exclusivement sur l'éducation lexicale : celui qui n'aurait pas appris que hépatocèle est une hernie partielle du foie ou que néphrite est une inflammation du rein ne saurait pas interpréter l'astérisque devant *hépatocèle du foie et *une néphrite au rein.

Je propose donc que la délimitation des domaines de la grammaire et du dictionnaire soit fonctionnelle, ou bien, selon la façon qu'a chacun d'accomplir ses tâches.

Si la langue est un système où tout se tient, la grammaire touche au lexique, étant donné que la langue fonctionne selon la grammaire et qu'elle ne fonctionne pas sans le lexique. Cela signifie que le dictionnaire doit tenir compte non seulement de l'information sémantique lexicale, mais aussi de l'information syntaxique et pragmatique qui permettent à l'élément de fonctionner. Ainsi, les informations grammaticales isolées et arbitraires que les dictionnaires de langue fournissent seront éliminées en faveur de l'élaboration d'entrées où ces informations seraient systématiques. C'est ainsi que chaque entrée se constitue en une mini-grammaire de l'élément, ce qui signifie qu'on a affaire à un dictionnaire fondé sur une théorie grammaticale (TG), qui assure son homogénéité et sa cohérence interne.

D'autre part, le dictionnaire se présentera comme une espèce de tableau pour la grammaire. Par exemple, l'identification correcte de la distribution de chaque mot est faite par le rapport entre ce mot et un autre de la même classe/sous-classe, et, par là, on aboutit à des règles générales de distribution qui se trouveront dans la grammaire. Et, encore, si pour chaque élément on indique à quelle classe/sous-classe il est associé, alors le dictionnaire apparaît comme un système de renvois et de corrélations contrôlé par la grammaire. Ainsi le dictionnaire et la grammaire ne se confondent ni ne s'excluent-ils pas, au contraire, ils se complètent. Le dictionnaire et la grammaire doivent être vus comme des parties d'un programme unifié de description linguistique dont le but principal serait l'identification de tous les phénomènes attachés à la fonction référentielle.

Cependant, un examen superficiel des dictionnaires de langue portugaise révèle que leur premier but est de lister des valeurs sémantiques (lexicales) soit par des équivalences, soit par des définitions/explications. On s'aperçoit qu'il n'y a pas de critères pour sélec- 
tionner ou, du moins, pour ordonner les informations. Par conséquent, il est très difficile de contrôler les incohérences et les redondances, ce qui peut compromettre la clarté et la rigueur des informations mises à jour dans le dictionnaire. La préoccupation première de l'information sémantique met en marge d'autres informations également utiles, selon le type de dictionnaire.

L'absence d'une conception sur la structure organisationnelle de la langue conduit à des remarques d'ordre épisodique, à des incohérences, à des fautes et à la subjectivité.

On saurait éviter ces écueils en adoptant une TG qui s'appliquerait aussi à la description sémantique.

On dira aussi que la description grammaticale n'a pas encore couvert tous les faits de langue, et qu'elle banalise les niveaux de langue en négligeant l'articulation entre langue et culture et, enfin que les dictionnaires, objets économiques, ne peuvent attendre que la grammaire soit assez accomplie pour répondre à un besoin social. Et puis, on ne peut pas attendre que l'usager soit un grammairien, évidemment. Mais il ne s'agit pas de mettre la grammaire partout, pas même en introduction des dictionnaires. Je fait référence à une conception grammaticale qui doit être sous-jacente à l'élaboration du dictionnaire.

Le défaut de la lexicographie traditionnelle est de présupposer de la compétence grammaticale du locuteur et du fait que la compétence lexicale n'est qu'une question d'éducation linguistique. Et c'est ainsi que le dictionnaire aurait pour but d'élucider et d'enrichir le lexique des gens, un procédé qui dure toute la vie. Cela étant, on dit que le dictionnaire n'est qu'un recueil de mots avec leurs significations. En général, le lexicographe ne s'occupe pas des difficultés des usagers au niveau de la syntaxe. Cela est un fait, d'ailleurs : tous les locuteurs, étrangers ou natifs, ont des difficultés liées à l'adéquation qui, à son tour, se rapporte à la distribution des unités.

Pour bien saisir le rôle joué par la grammaire dans l'organisation d'un dictionnaire d'usages, il est bon de ne pas perdre de vue que le dictionnaire, en tant qu'outil pédagogique, traduit un ensemble d'attentes de la communauté à laquelle il s'adresse. C'est pour cela, peut-être, que Weinrich dit que la vérité des dictionnaires est une vérité historique, qui change d'une époque à l'autre. Par exemple, les dictionnaires des académies du XVIIe siècle reflètent une préoccupation normative d'après les modèles classiques: le bon usage décidé par le jugement des savants. Ces dictionnaires enregistrent et contrôlent ce qui doit être dit. Ils diffèrent beaucoup des dictionnaires du $\mathrm{XX}^{\mathrm{e}}$ siècle, d'une part, par leur conception propre, et, d'autre part, parce qu'on dirait qu'ils sont l'esprit d'une époque. Les objectifs conditionnent l'encadrement des entrées. Les dictionnaires anciens, dans la mesure où ils fixaient les normes du parler et de l'écrit à partir de l'autorité des modèles transmis par la tradition écrite, ne dégageaient pas d'exemples: le témoin des auteurs «consacrés». Aujourd'hui, on enregistre encore l'usage parlé. La langue écrite a toujours, évidemment, incorporé les formes du parler; aujourd'hui, cela se fait plus directement et plus vite, c'est-à-dire moins sélectivement, de telle façon que l'on peut considérer comme neutres, selon qu'ils appartiennent à un niveau ou à un autre, les textes non marqués par des traits littéraires ou techniques: les pièces théâtrales, les scripts de cinéma, les feuilletons de télévision sont, en principe, une transposition en direct de la langue parlée.

Notre époque possède un très grand outillage technologique. La science fait de plus en plus partie de la vie sociale et privée, en déterminant les conditions de l'ambiance où nous sommes obligés de vivre. Le dictionnaire est donc vu comme un outil de travail où s'accentue la langue dans sa fonction d'interaction sociale. On bénéficie du progrès technologique qui permet des résultats des plus objectifs. De nos jours, il est très facile de constater/observer comment on dit, à travers une simple comparaison des moyennes d'usage, des données qu'un ordinateur du type PC offre aisément. 
Ainsi, les attentes vis-à-vis de l'utilité des dictionnaires sont-elles aujourd'hui différentes du passé. Par exemple, on consulte souvent un dictionnaire pour chercher le sens d'un mot, mais pas nécessairement pour connaître son usage correct d'après les normes traditionnelles. Et c'est, il me semble, la technologie qui motive la circulation des sens : le poids sémantique spécifique des termes techniques répandus par les journaux et par les magazines échappe à l'usager. Étant donné que le mot ne fonctionne que dans un contexte typique, où il a sa juste valeur, il faut, d'abord, décrire ces contextes-là, c'est-à-dire révéler comment les mots fonctionnent, ce qui ne devient possible que par le biais d'une $\mathrm{TG}$, car, si l'on veut employer effectivement le mot qu'on cherche, il faut, au minimum, observer sa distribution, ce qui est déjà une propriété syntaxique.

Quant à l'information syntaxique, la valeur d'un dictionnaire d'usages est due, en partie, à la fonction du nombre des phrases à différentes structures qu'il associe à chaque entrée.

Pour saisir la langue, il faut d'abord identifier les rapports entre les phrases par des paraphrases ou des transformations. L'établissement des équivalences et des transformations a une première conséquence: l'écart de la notion de partie du discours comme fondement effectif de l'analyse grammaticale. C'est une telle position qui justifie l'élaboration d'une lexicogrammaire, un outil pour améliorer l'organisation du dictionnaire en tant qu'objet de régularité, d'économie et de rigueur. Par exemple, les prépositions sont entrées dans nos dictionnaires de façon très irrégulière. Le choix de quelques valeurs sémantiques générales est très courant (ex.: mode, temps, causatif, lieu), ce qui aboutit à une classification pour les emplois individuels. En général, on ne s'occupe pas du poids grammatical de la préposition dans la langue en question. On constate aussi ce vide pour les mots grammaticaux en général.

Un des problèmes du lexicographe est l'exhaustivité dans la description des emplois. Une description par des matrices constructionnelles, par exemple, permettrait de contrôler l'usage en signalant les erreurs (*).

L'absence d'une TG à des indications syntaxiques à l'intérieur des informations d'ordre sémantique, à des informations indirectes et non systématiques. C'est une orientation grammaticale systématique et exacte qui permet l'élaboration du dictionnaire à traits spécifiques et à objectifs bien définis. Il ne s'agit pas de réfuter, et pas même d'annuler un travail considérable et de longue haleine pour réunir un ensemble très important de renseignements sur le vocabulaire de la langue.

Si un dictionnaire est un objet économique et, surtout, pédagogique, plus il sera rigoureux et cohérent, plus grande sera son utilité. 